\title{
Green Synthesis of Ag 1D Nanochains and Application for Methylene Blue Degradation
}

\author{
Karina Chavez and Gerardo Rosas
}

Universidad Michoacana de San Nicolás de Hidalgo, Morelia, Michoacan de Ocampo, Mexico

Introduction.

The biosynthesis of nanoparticles is a representative intersection between nanotechnology and biotechnology, has increased attention due to the growing need to develop environmentally benign technologies in material synthesis [1]. Such nanoscale materials possess unique electrical, optical as well as biological properties and are thus applied in catalysis, biosensing, imaging, drug and delivery [2-3]. Silver nanoparticles are essential materials that have been studied extensively, especially the formation of anisotropic 1D arrays by the association of their collective properties [4]. The "bottom-up" synthesis approach of nanomaterials first forms the nanostructured units, and then assembles to obtain the final material [5]. In nature, biomolecules participate to form total self-assembly. Therefore, self-assembly using a green approach can depend either on the characteristics of the plant extract [6]. In this work, we present a facile strategy for the green synthesis of silver nanochains using the Hamelia patens plant extract as reducing and capping agent, that is achieved through the self-assembly into a colloidal solution and at room temperature conditions. Also, the efficiency of silver nanochains was evaluated for the catalysis of an organic dye.

\section{Methods.}

The extract was prepared using $2 \% \mathrm{w} / \mathrm{v}$ concentration of Hamelia patens leaves in $50 \mathrm{ml}$ of deionized water, and then the solution was heated to $50^{\circ} \mathrm{C}$, under magnetic stirring for $15 \mathrm{~min}$. The reaction solution was filtered using a filter paper. The precursor was prepared in aqueous solution at $12 \mathrm{mM} \mathrm{AgNO}_{3}$ ( $\geq 99.9 \%$ ). Silver nanostructures were prepared by adding the aqueous extract from the plant to the precursor, using a volumetric ratio of 2:1 at room temperature. The catalytic activity of Ag nanochains was evaluated without any catalyzer for the degradation of $0.05 \mathrm{mM}$ methylene blue (MB). $0.5 \mathrm{mg}$ of nanostructures $(12 \mathrm{mM})$ were mixed with $2.5 \mathrm{ml}$ of $\mathrm{MB}$. The morphology was evaluated by scanning electron microscopy (SEM) in a JEOL JSM-7600F microscope with an energy dispersive spectrometer (EDS). Philips Tecnai F20 microscope operated at an accelerating voltage of $200 \mathrm{kV}$ was used for transmission electron microscopy (TEM). UV-vis absorption spectra was obtained using an Ocean- Optics USB4000 spectrometer in the range of 300-700 nm.

Results.

The nanostructures synthesized with the Hamelia patens plant were characterized by scanning electron microscopy (SEM) (Fig. 1a-b). In the micrographs are observed linked particles as chains-like, a higher magnification illustrates that the structures are inside the nanometric range, with average sizes of $20 \mathrm{~nm}$. The chemical analysis performed by EDS (Fig. 1c), verifies that the particles are formed by silver, $\mathrm{Cu}$ appears which comes from the sample holder. The high-resolution transmission electron microscopy (HRTEM) shows nanochains with $18 \mathrm{~nm}$ particle size. The nanoparticles are linked on the close-packed plane (111), as illustrated in the FFT (insert).

In this study, the color of the reaction mixture changes from yellow to dark brown in 5 min, indicating the presence of the silver nanomaterials. The nanometer nature of the silver can be determined by the Uv-vis technique since only nanometer sizes are registered due to quantum confinement. Figure 2 a shows the Uv- 
vis spectra of the silver nanochains synthesized. The characteristic absorption band of the silver nanoparticles is observed between 400 and $500 \mathrm{~nm}$, the surface resonance plasmon (SPR), it is monitored each $15 \mathrm{~min}$, and as the time increases, the absorption increases and the peak widens. Figure $2 \mathrm{~b}$ shows the UV-vis spectra corresponding to the catalytic properties of the Ag-nanochains, for the degradation of methylene blue (MB) at room temperature conditions, the reaction was evaluated without catalyst (i.e., $\mathrm{NaBH} 4$ ). From the curves, it can observe that the intensity of the dye decrease as the redox reaction progresses. It was found that the nanomaterial showed a 90\% dye degradation obtained after $150 \mathrm{~min}$. This result indicates that the silver nanostructures can be used in the removal of organic dyes in residual water. In other investigations [7-8], they used silver nanoparticles with a catalyst $\left(\mathrm{NaBH}_{4}\right)$ to accelerate the degradation of MB. However, to know the real effect of the nanochains in this work, it was not used as a catalyst.

Conclusions

In summary, 1D silver nanoarrays can be obtained using the Hamelia patens plant extract. The SEM micrographs show silver nanochains-like with a uniform distribution. The TEM analysis showed that the planes through which the nanoparticles are linked to form the chains are (111) and that the particle sizes are $18 \mathrm{~nm}$. In addition, Ag nanochains show 90\% degradation of MB after $150 \mathrm{~min}$.

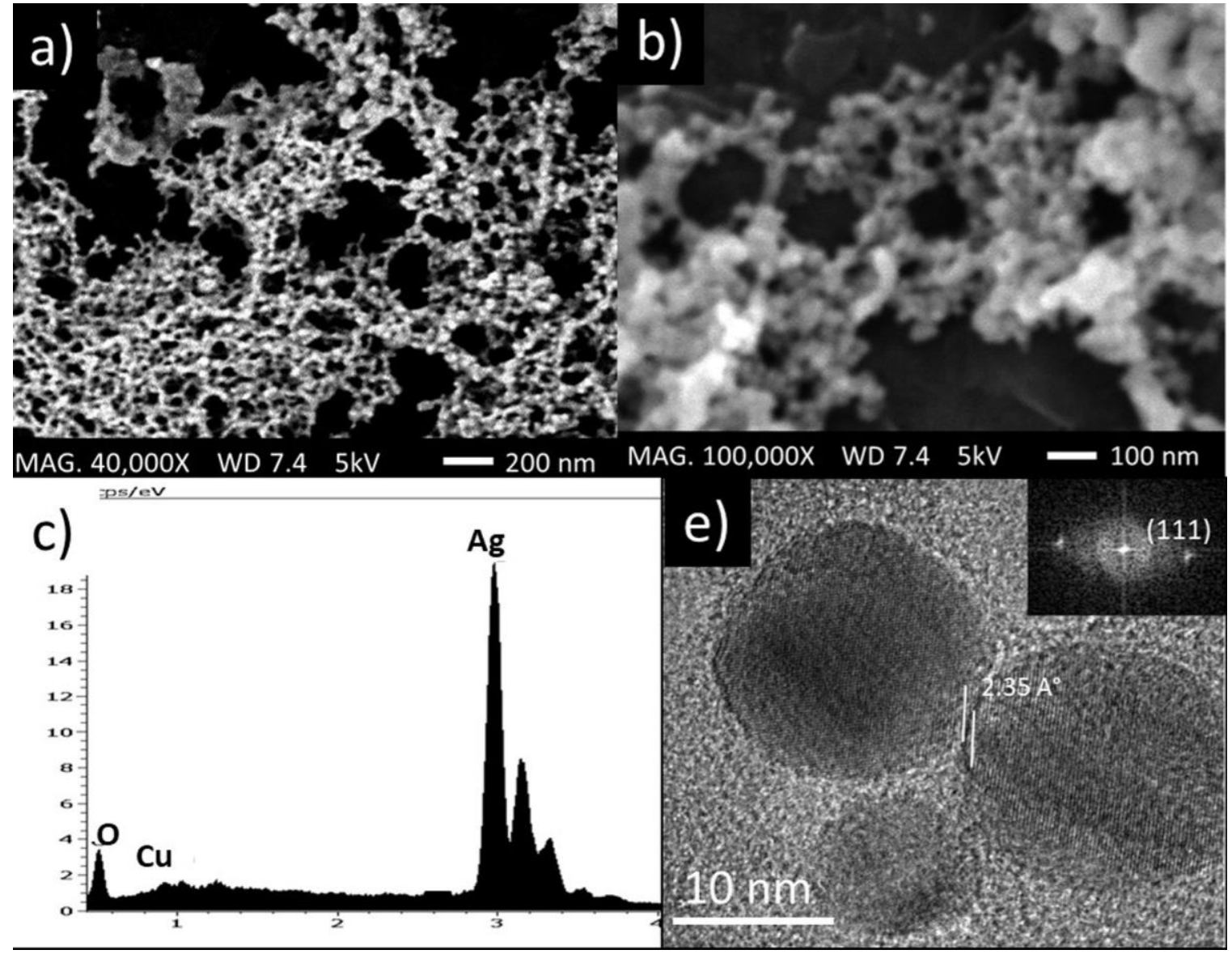

Figure 1. Figure 1. SEM images of Ag nanochains (a) 40,000X, (b) 100,000X, (c) EDS and (d) HRTEM. 


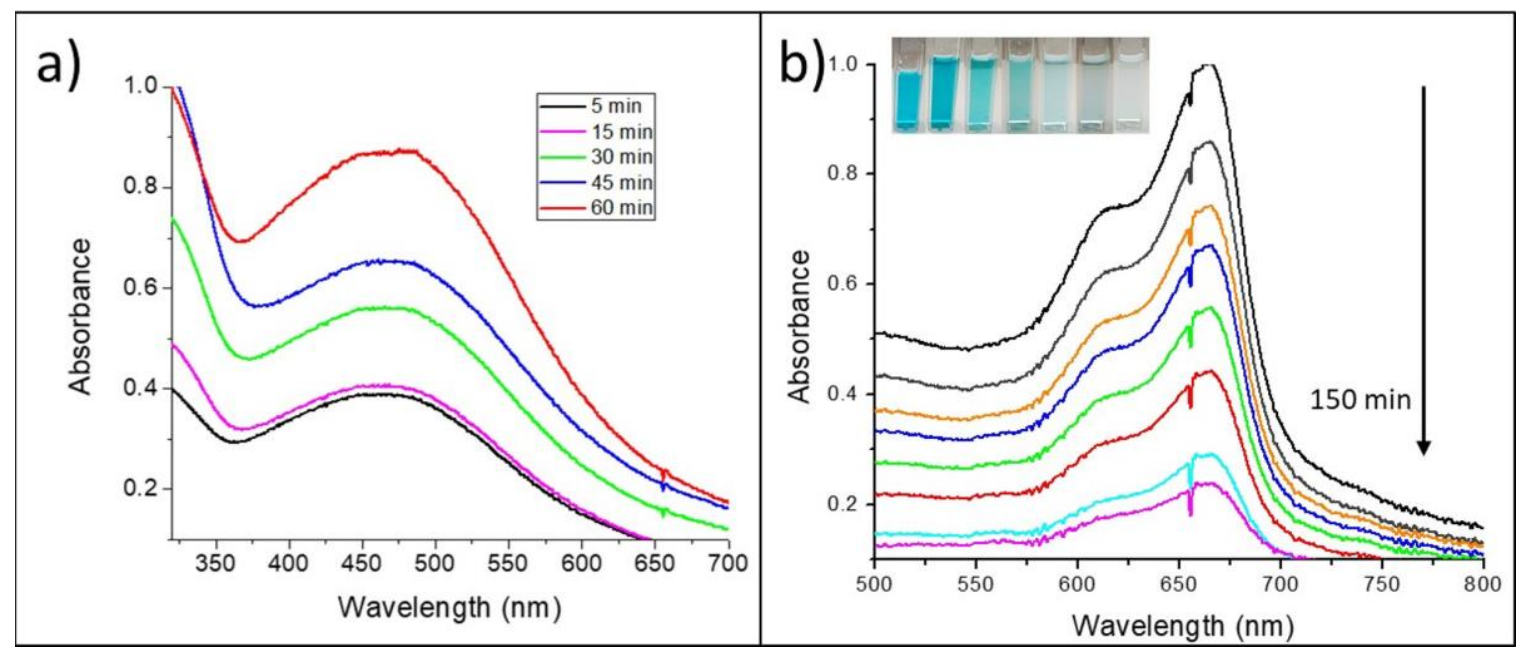

Figure 2. Figure 2. UV-visible spectra of (a) silver nanochains and (b) MB reduction in the presence of synthesized Ag-nanochains.

\section{References}

[1] Sathishkumar, M., Sneha, K., Won, S. W., Cho, C. W., Kim, S., \& Yun, Y. S. (2009). Cinnamon zeylanicum bark extract and powder mediated green synthesis of nano-crystalline silver particles and its bactericidal activity. Colloids and Surfaces B: Biointerfaces, 73(2), 332-338. [2] Gajanan, K., \& Tijare, S. N. (2018). Applications of nanomaterials. Materials Today: Proceedings, 5(1), 1093-1096. [3] Rasmussen, K., Rauscher, H., Mech, A., Sintes, J. R., Gilliland, D., González, M., ... \& Bleeker, E. A. (2018). Physico-chemical properties of manufactured nanomaterials-Characterisation and relevant methods. An outlook based on the OECD Testing Programme. Regulatory Toxicology and Pharmacology, 92, 8-28. [4] García, M. C., Aloisio, C., Onnainty, R., \& Ullio-Gamboa, G. (2018). Selfassembled nanomaterials. In Nanobiomaterials (pp. 41-94). Woodhead Publishing. [5] Arole, V. M., \& Munde, S. V. (2014). Fabrication of nanomaterials by top-down and bottom-up approaches-an overview. J. Mater. Sci, 1, 89-93. [6] Shin, Y., Bae, I. T., \& Exarhos, G. J. (2009). "Green" approach for self-assembly of platinum nanoparticles into nanowires in aqueous glucose solutions. Colloids and Surfaces A: Physicochemical and Engineering Aspects, 348(1-3), 191-195. [7] Saha, J., Begum, A., Mukherjee, A., \& Kumar, S. (2017). A novel green synthesis of silver nanoparticles and their catalytic action in reduction of Methylene Blue dye. Sustainable Environment Research, 27(5), 245-250. [8] Bonnia, N. N., Kamaruddin, M. S., Nawawi, M. H., Ratim, S., Azlina, H. N., \& Ali, E. S. (2016). Green biosynthesis of silver nanoparticles using 'Polygonum Hydropiper' and study its catalytic degradation of methylene blue. Procedia Chem, 19, 594-602. 